\title{
Continuous Selection of the Fastest Growing Species in the Chemostat
}

\author{
Pierre Masci, Olivier Bernard, and Frédéric Grognard \\ INRIA-Project COMORE, 2004 route des lucioles, BP 93, 06902 \\ Sophia Antipolis (Cedex), FRANCE \\ Tel: 0033.4.92.38.71.85; e-mail: pierre.masci@sophia.inria.fr, \\ olivier.bernard@sophia.inria.fr,frederic.grognard@sophia.inria.fr
}

\begin{abstract}
This paper proposes closed loop controls for microorganisms in the chemostat, which make it possible to select species with fastest growth rate in chosen environmental conditions. In particular, by controlling the dilution rate and the input substrate concentration, it is possible to select a species which maximizes a criterion. In a first step a control is proposed for Monod's and Droop's models in order to regulate the total biomass concentration. We show that this causes the selection of the fastest growing species if the system has a periodic behavior. Then we propose a way of achieving periodic stresses, and of selecting the species with maximal potential growth rate. Finally, the method is simulated using Droop's model for selecting a species which can both grow fast and increase its internal substrate storage.
\end{abstract}

Keywords: chemostat; competition; microorganisms; control

\section{INTRODUCTION}

The chemostat is an open bioreactor where a microorganism can grow in suboptimal conditions of substrate limitation. The chemostat model supports several ecological theories (S.R. Hansen and S.P. Hubell [1980] - R. Arditi and H. Saiah [1992] - B.J.M. Bohannan and R.E. Lenski [2000]) that were then extrapolated and tested in real life. Among these theories, the competition theory is one of the most famous. It states that if $n$ competing species are introduced in a chemostat, generically (in the adequate working modes) only one species will stay in the chemostat, while the $n-1$ other will disappear. This principle was validated with real experiments in S.R. Hansen and S.P. Hubell [1980] where the species that "wins" the competition could be predicted; it was the one that can grow at a constant rate (equal to the dilution rate as detailed further on) with the smallest amount of limiting nutrient $s^{*}$. This theoretical result could be used to select among a blend of species, the ones of interest. This idea was first introduced by S.W. Brown and S.G. Oliver [1982] and then by Juan Jimnez and Taha Bentez [1988], who have made chemostat experiments for selecting ethanoltolerant mutants. By adjusting a particular stress such as an inhibitor's concentration, a substrate limitation, or the dilution rate, it can lead to the isolation of species with optimal yield. This is of particular importance since it is a rather simple way of making a particular species emerge within a population. However, in most biotechnological applications, the selection criterion based on the idea of "optimal yield" is not appropriate. A selection that would e.g. select the microorganism with the highest growth potential would be preferable, especially if one wants to identify organisms that grow in hostile conditions. More generally, the objective of this paper is to propose new selection criteria. For this, the chemostat is not run in open loop, but a control law is proposed to run the system in closed loop. We show how the competition outcome is modified and we propose new criteria that could be used for species separation.

The paper is structured as follows. In a first part we recall two classical models of microorganisms in the chemostat, and the classical competition principle. In section 2 we study a first control law that puts the chemostat into a turbidostat mode, and we show that this control causes the selection of the fastest growing species if the system has a periodic behavior. Finally we propose a control to achieve periodic substrate stresses. In section 4 we maintain a periodical substrate limitation. A simulation example illustrates the benefit of the approach and shows how three species can be separated on this principle.

\section{SHORT REVIEW OF COMPETITION ON A SINGLE SUBSTRATE IN THE CHEMOSTAT}

\subsection{Basic model for microorganisms growing in the chemostat}

Monod's model (1) is the basic model for describing microorganisms' growth on a single substrate in a chemostat.

$$
\left\{\begin{array}{l}
\dot{s}=D\left(s_{i n}-s\right)-\sum_{i=1}^{N} \frac{1}{y_{i}} \mu_{i}(s) x_{i} \\
\dot{x}_{i}=\left(\mu_{i}(s)-D\right) x_{i}
\end{array}\right.
$$

where $s$ stands for the substrate concentration in the chemostat, $s_{i n}$ its input concentration, and $x_{i}$ the $i$ th species biomass concentration. The $\mu_{i}$ functions represent the growth rates of these species, and the $y_{i}$ constants are their biomass/substrate conversion yields. $D$ is the input/output dilution rate. The system can be controlled with $s_{i n}$ and $D$. 
In this model the growth rate functions $\mu_{i}$ are taken as positive monotonic increasing functions

$$
s_{a}<s_{b} \Leftrightarrow \mu_{i}\left(s_{a}\right)<\mu_{i}\left(s_{b}\right)
$$

They are bounded by their supremum values $\mu_{m_{i}}$ :

$$
\mu_{i}(s)<\mu_{m_{i}} \quad \text { and } \quad \lim _{s \rightarrow+\infty} \mu_{i}(s)=\mu_{m_{i}}
$$

\subsection{Variable yield model}

Droop's model (4) is more complex but describes the internal substrate storage $q$ of the microorganisms. It is more appropriate to describe microalgae's growth.

$$
\left\{\begin{array}{l}
\dot{s}=D\left(s_{i n}-s\right)-\sum_{i=1}^{N} \rho_{i}(s) x_{i} \\
\dot{q}_{i}=\rho_{i}(s)-\mu_{i}\left(q_{i}\right) q_{i} \\
\dot{x}_{i}=\left(\mu_{i}\left(q_{i}\right)-D\right) x_{i}
\end{array}\right.
$$

In this model the $\rho_{i}$ functions represent the substrate absorption rates, while the $\mu_{i}$ functions represent the growth rates. These functions are positive monotonic increasing functions. They are bounded by the supremum values $\rho_{m_{i}}$ and $\bar{\mu}_{i}$.

For each fixed substrate concentration $s$, the $\dot{q}_{i}$ equation indicates that $q_{i}$ goes towards $Q_{i}(s)$, defined as the unique solution of

$$
\mu_{i}\left(Q_{i}(s)\right) Q_{i}(s)=\rho_{i}(s)
$$

Indeed, the uniqueness of $Q_{i}(s)$ and its attractivity are straightforward since $\mu_{i}\left(q_{i}\right) q_{i}$ is increasing in $q_{i}$.

Let us define the maximum internal substrate storage

$$
Q_{m_{i}}=\lim _{s \rightarrow+\infty} Q_{i}(s)
$$

$Q_{m_{i}}$ is thus the solution of $\mu_{i}\left(Q_{m_{i}}\right) Q_{m_{i}}=\rho_{m_{i}}$. It defines an upper-bound for $q_{i}$ along the solutions of (4). Indeed, if $q_{i}=Q_{m_{i}}$, then $\dot{q}_{i}=\rho_{i}(s)-\rho_{m_{i}}<0$, and if $q_{i}<Q_{m_{i}}$ is verified at the initial time, then it is always verified. The fact that there is a maximum internal substrate storage $Q_{m_{i}}$ for each species is biologically relevant.

Corresponding to this maximum internal substrate storage, there exists a maximum growth rate $\mu_{m_{i}}$ for each species

$$
\mu_{m_{i}}=\mu_{i}\left(Q_{m_{i}}\right)=\frac{\rho_{m_{i}}}{Q_{m_{i}}}
$$

\subsection{The Competitive Exclusion Principle (CEP)}

Under constant controls $D$ and $s_{i n}$, for some species, there exists a substrate concentration $s_{i}^{\star}$ for which the growth rate $\mu_{i}$ is (or becomes) equal to the dilution rate $D$ :

$$
\begin{aligned}
& \mu_{i}\left(s_{i}^{\star}\right)=D \quad \text { in Monod's model } \\
& \mu_{i}\left(Q_{i}\left(s_{i}^{\star}\right)\right)=D \quad \text { in Droop's model }
\end{aligned}
$$

If this substrate concentration does not exist for a given species, it means that $\mu_{i}(s)<D \quad \forall s$ (or $\mu_{i}\left(Q_{i}(s)\right)<$ $D \quad \forall s$ in Droop's model) and the species will be washed out of the chemostat. If $\mu_{i}\left(s_{i n}\right)$ (or $\left.\mu_{i}\left(Q_{i}\left(s_{i n}\right)\right)<D\right)$ for a particular species, that species will also be washed out of the chemostat because we will have $s(t)<s_{\text {in }}$ after some finite time $t_{0}$, and then $\mu_{i}(s(t))$ or $\mu_{i}\left(Q_{i}(s(t))\right)<$ $\mu_{i}\left(s_{i n}\right)<D \quad$ for all $t>t_{0}$

The CEP stipulates that in a chemostat with $\mathrm{N}$ species that satisfy (8) (so that they are not guaranteed to be washed out), single substrate growth limitation, and constant controls $D$ and $s_{i n}$, all but one of the species converge to 0 :

$$
\begin{aligned}
& \text { If } \exists n \in\{1,2, \ldots, N-1, N\} \text { so that } s_{n}^{\star}<s_{i}^{\star} \quad \forall i \neq n \\
& \text { then } \lim _{t \rightarrow+\infty} x_{i}(t)=0 \quad \forall i \neq n
\end{aligned}
$$

Criterion 1. CEP's Competitiveness Criterion

The species with smallest $s_{i}^{\star}$, who needs less substrate than the others for reaching a growth rate $\mu_{i}(s)$ equal to the dilution rate $D$, wins the competition and excludes all other species from the chemostat.

This result can be found in H.L. Smith and P. Waltman [1995] for the Monod model with $\mathrm{N}$ species and for the Droop model with 2 species, and validated with several species (S.R. Hansen and S.P. Hubell [1980] - H. Ducobu, J. Huisman, R.R. Jonker, and L.R. Mur [1998] J. Passarge, S. Hol, M. Escher, and J. Huisman [2006]).

The CEP is crucial for the understanding of natural ecosystems. That is why so many people discussed it. Some (B.T. Li , H.L. Smith [2003] - C. Lobry, F. Mazenc, and A. Rapaport [2005] - J. Hesseler, J.K. Schmidt, U. Reichl, D. Flockerzi [2006] - C. Lobry and J. Harmand [2006]) developped alternative models for which the CEP is not verified, in order to explore some coexistence cases. S.S. Pilyugin, G.T. Reeves, and A. Narang [2004] worked on multiple substrate limitations, and showed in these conditions that it is possible to infer the result of competition from the dynamics of single species cultures.

Some works have already been done to control the competition in the chemostat, generally to enable the coexistence of several species (N.S. Rao and E.O. Roxin [1990] - P. de Leenheer, B. Li, and H.L. Smith [2003] - J. Gouzé and G. Robledo [2005]). What we aim at doing here is to find controls which change the result of the competition keeping a single species. More precisely, we want to select species of interest who maximize a criterion other than the smallest $s_{i}^{\star}$, by imposing a periodic behaviour to the system.

O. Bernard, G. Malara, and A. Sciandra [1996] have studied the effect of periodic substrate stresses, which is a realistic ecological situation. In section 4 we have determined controls which permit to reproduce such stresses, and in section 5.2 we have shown that this causes a new selection criterion other than Criterion 1.

\section{SELECTING THE FASTEST GROWING SPECIES}

\subsection{Control of the total biomass concentration}

For selecting such a species in the chemostat, we need to regulate the total biomass concentration $X_{T}=\sum_{i=1}^{N} x_{i}$ so that the chemostat does not become saturated with microorganisms. We achieve this by controlling $D$ so that

$$
\dot{X}_{T}=D\left(X_{T}^{\star}-X_{T}\right)
$$


In both models this leads us to $\sum_{i=1}^{N} \mu_{i}(.) x_{i}-D X_{T}=$ $D\left(X_{T}^{\star}-X_{T}\right)$ and

$$
D=\sum_{i=1}^{N} \frac{x_{i}}{X_{T}^{\star}} \mu_{i}(.)
$$

When $X_{T}$ is close to $X_{T}^{\star}, D$ is the weighted mean of the growth rates $\mu_{i}$ of the microorganisms.

It is also possible to regulate $K_{T}=\sum_{i=1}^{N} k_{i} x_{i}$ with $k_{i}$ coefficients who can represent turbidity coefficients. This is what is approximatively done in a particular family of chemostat, the turbidostat.

\subsection{The fastest species excludes all others from the} chemostat

For this study the growth functions in both models will be considered only time-dependent functions $\mu_{i}(t)$, with $T_{i}$-periodic behaviors. As we shall later see such periodic behaviors can be obtained by different means. We use the following notation for the mean growth of the species :

$$
\mu_{\text {mean }_{i}}=\frac{1}{T_{i}} \int_{0}^{T_{i}} \mu_{i}(t) d t
$$

Hypothesis 2. Let us assume that

$\exists n \in[1 ; N]$ so that $\mu_{\text {mean }_{n}}>\mu_{\text {mean }_{i}} \quad \forall i \neq n$

Theorem 3. Selection for any periodic behavior

With a bounded total biomass concentration $X_{T}=$ $\sum_{i=1}^{N} x_{i}$, species $n$ with biggest mean growth $\mu_{\text {mean }}$ is selected : it excludes all other species from the chemostat.

Proof We denote $d_{i}=\ln \left(\frac{x_{n}}{x_{i}}\right)$

With $\dot{x}_{i}=\left(\mu_{i}-D\right) x_{i}$ we obtain

$$
\begin{aligned}
\dot{d}_{i}(t) & =\frac{\dot{x}_{n}(t)}{x_{n}(t)}-\frac{\dot{x}_{i}(t)}{x_{i}(t)}=\mu_{n}(t)-\mu_{i}(t) \text { and } \\
d_{i}(t) & =d_{i}(0)+\int_{0}^{t} \dot{d}_{i}(\tau) d \tau \\
& =d_{i}(0)+\int_{0}^{t} \mu_{n}(\tau) d \tau-\int_{0}^{t} \mu_{i}(\tau) d \tau
\end{aligned}
$$

We then use the following notations :

$$
\begin{aligned}
& p_{i}(t)=\frac{t-t \equiv T_{i}}{T_{i}} \\
& r_{i}(t)=t \equiv T_{i} \\
& t=p_{i}(t) T_{i}+r_{i}(t)
\end{aligned}
$$

where $t \equiv T_{i}$ represents tmodulo $T_{i}$, and $p_{i}(t)$ is the number of $T_{i}$-periods for species $i$ until time $t$. which leads us to

$$
\begin{aligned}
& \int_{0}^{t} \dot{d}_{i}(\tau) d \tau=p_{n}(t) \int_{0}^{T_{n}} \mu_{n}(\tau) d \tau-p_{i}(t) \int_{0}^{T_{i}} \mu_{i}(\tau) d \tau+R_{i}^{n}(t) \\
& =p_{n}(t)\left(\int_{0}^{T_{n}} \mu_{n}(\tau) d \tau-\frac{p_{i}(t)}{p_{n}(t)} \int_{0}^{T_{i}} \mu_{i}(\tau) d \tau\right)+R_{i}^{n}(t) \\
& =p_{n}(t)\left(T_{n} \mu_{\text {mean }_{n}}-\frac{p_{i}(t)}{p_{n}(t)} T_{i} \mu_{\text {mean }_{i}}\right)+R_{i}^{n}(t) \\
& \text { with notation } R_{i}^{n}(t)=\int_{0}^{r_{n}(t)} \mu_{n}(\tau) d \tau-\int_{0}^{r_{i}(t)} \mu_{i}(\tau) d \tau
\end{aligned}
$$

and with $\frac{p_{i}(t)}{p_{n}(t)}=\frac{t-t \equiv T_{i}}{t-t \equiv T_{n}} \frac{T_{n}}{T_{i}}$, since $\lim _{t \rightarrow+\infty} \frac{t-t \equiv T_{i}}{t-t \equiv T_{n}}=1$, and $d_{i}(0)$ and $R_{i}^{n}(t)$ are bounded, $\lim _{t \rightarrow+\infty} d_{i}(t)=+\infty$ because $\lim _{t \rightarrow+\infty} p_{i}(t)=+\infty$ and $\mu_{\text {mean }_{n}}>\mu_{\text {mean }_{i}}$

Thus $\lim _{t \rightarrow+\infty} \frac{x_{n}(t)}{x_{i}(t)}=+\infty$ and, as $x_{n}$ is upper bounded by the upper-bound on $X_{T}$, then $\lim _{t \rightarrow+\infty} x_{i}(t)=0$ and the proof is complete.

\subsection{Selection of the species with biggest $\mu_{m_{i}}$}

We have shown that it is possible to select a species with fastest growth in given environmental conditions. Here we try to determine conditions which will allow us to select the species with biggest $\mu_{m_{i}}$.

Criterion 4. $\mu_{m_{i}}$ Competitiveness Criterion

With a bounded total biomass concentration $X_{T}=$ $\sum_{i=1}^{N} x_{i}$, and with $s$ big enough so that $\mu_{i}(t) \approx \mu_{m_{i}} \quad \forall t$, the species with fastest growth $\mu_{m_{i}}$ is selected : it excludes all other species from the chemostat.

\section{Proof and effective realization}

The demonstration of Criterion 4 is the same as for Theorem 3 , with $\mu_{i}(t)=\mu_{m_{i}}$.

In order to have $\mu_{i}(t) \approx \mu_{m_{i}}$, we regulate $s$ at a large value $s_{0}$ (so that $\mu_{i}\left(s_{0}\right)$ or $\left.\mu_{i}\left(Q_{i}\left(s_{0}\right)\right) \approx \mu_{m_{i}}\right)$ by imposing

$$
\dot{s}=D\left(s_{0}-s\right)
$$

which is achieved through $s_{i n}=s_{0}+\frac{1}{D} \sum_{i=1}^{N} \rho_{i}(s) x_{i}$

As $\sum_{i=1}^{N} \rho_{i}(s) x_{i}$ is not always measurable, we have imagined a simpler way to realize $\mu_{i}\left(Q_{i}(s)\right) \approx \mu_{m_{i}}$ : with a big constant $s_{i n}$ and a small $X_{T}^{\star}$ we have $\dot{s} \approx D\left(s_{i n}-\right.$ $s)$ and the substrate concentration will converge close to $s_{i n}$, which is chosen big enough to have $\mu_{i}\left(s_{i n}\right) \approx \mu_{m_{i}}$ $\left(\mu_{i}\left(Q_{i}\left(s_{i n}\right)\right) \approx \mu_{m_{i}}\right.$ in Droop's model $)$.

\section{ACHIEVING PERIODIC SUBSTRATE STRESSES}

We propose an approach for the generation of a periodic behavior in the case of Droop's model, under control $D$ (11). It consists in approximating an ideal T-periodic behavior of interest. In the Droop model for instance, we have reproduced a situation of periodic substrate stresses, with the following T-periodic shape for $\rho_{i}(s)$ :

$$
s(t)= \begin{cases}s_{M} & \text { for } t \equiv T \leq \tau \\ 0 & \text { for } t \equiv T>\tau\end{cases}
$$

with $s_{M}$ such that $\rho_{i}\left(s_{M}\right) \approx \rho_{m_{i}}$. The ratio $\tau / T$ is the proportion of time the microorganisms are fed, and $(T-\tau) / T$ is the proportion of time during which a stress is imposed to the microorganisms. Such a shape can be approached as shown in Figure 1, with two steps : a rising phase and a falling one. The approximation is good if $s(t) \approx s_{M}$ for most of the time interval $[0, \tau]$.

Rising phase during time $\tau_{\text {rise }}$

We choose $s_{i n}$ such that $\dot{s}=\lambda\left(s_{M}-s\right)$ :

$$
s_{i n}=s+\frac{1}{D}\left(\lambda\left(s_{M}-s\right)+\sum_{i=1}^{N} \rho_{i}(s) x_{i}\right)
$$




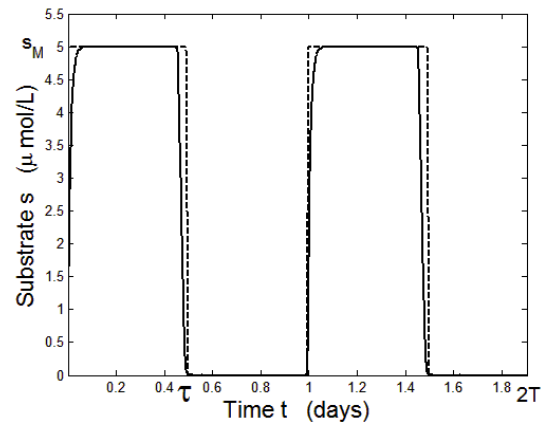

Fig. 1. Approaching ideal periodic substrate stresses (15) (dashed), with a rising and a falling phase (solid)

There is no positivity problem for $s_{\text {in }}$ here because $s_{M}>s$. Then $s(t)=s_{M}\left(1-e^{-\lambda t}\right)$ with $s(0)=0$, and with a big $\lambda$ we obtain $s(t) \approx s_{M}$ for most of the time interval $\left[0, \tau_{\text {rise }}\right]$

Falling phase during time $\tau_{\text {fall }}=\tau-\tau_{\text {rise }}$

We choose $s_{i n}$ such that $\dot{s}=-\gamma \phi(s)$, with $\phi(s)<$ $\rho_{i}(s) \quad \forall i \in\{1, \ldots, N\}, \forall s \in\left[0, s_{M}\right]$

$$
s_{i n}=s+\frac{1}{D}\left(X_{T}\left(\sum_{i=1}^{N} \frac{x_{i}}{X_{T}} \rho_{i}(s)\right)-\gamma \phi(s)\right)
$$

Since $\sum_{i=1}^{N} \frac{x_{i}}{X_{T}} \rho_{i}(s)$ is a convex combination of the $\rho_{i}(s)$, we have for all $s: X_{T} \sum_{i=1}^{N} \frac{x_{i}}{X_{T}} \rho_{i}(s) \geq X_{T} \min _{i} \rho_{i}(s)>$ $X_{T} \phi(s)$, so that $s_{i n}>0$ if $X_{T}>\gamma \forall t$ For having $s_{i n}>0$ we need $\phi(s) \gamma<\left(\sum_{i=1}^{N} \frac{x_{i}}{X_{T}} \rho_{i}(s)\right) X_{T}$. This can be achieved with $\gamma<\min \left(X_{T}^{\star}, X_{T}(0)\right)$ under control (11).

Finally, as we will see in section 5 , it is possible to estimate the falling time $\tau_{\text {fall }}$ with $\frac{d s}{d t}=-\gamma \phi(s)$, and to obtain a periodic $s$ which permits us to approach control (15). By choosing $X_{T}^{\star}$ big enough and waiting for $X_{T}$ to be close to $X_{T}^{\star}$ before starting the $s_{i n}$ control, $\gamma$ can be chosen big and $s(t) \approx 0$ is reached fast.

Thus, controls (16) and (17) permit us to achieve periodic behavior near (15) for $s(t)$.

With control (15) we get approximately the following $\dot{q}_{i}$ :

$$
\begin{array}{r}
\dot{q}_{i}=\rho_{i}-\mu_{i}\left(q_{i}\right) q_{i} \\
\text { with } \rho_{i}(t)= \begin{cases}\rho_{m_{i}} & \text { for } t \equiv T \leq \tau \\
0 & \text { for } t \equiv T>\tau\end{cases}
\end{array}
$$

and it is now possible to show that

Theorem 5. There exists a unique periodic solution $\bar{q}_{i}(t)$ to (18). This periodic solution is attractive for any initial condition $q_{i}(0)$ inside $\left[0, Q_{m_{i}}\right]$

Proof In the case $q_{i}(0)=0$ we have $q_{i}(\tau)>0$ because $\mu_{i}\left(q_{i}\right) q_{i}<\rho_{m_{i}}$, and $q_{i}(\tau)>0$ implies $q_{i}(T)>0$ because $\mu_{i}\left(q_{i}\right) q_{i}<\mu_{m_{i}} q_{i}$ and the decrease is slower than an exponential decrease with time constant $\mu_{m_{i}}$. With $q_{i}(0)=0$ we obtain $q_{i}(T)>q_{i}(0)$.

In the case $q_{i}(0)=Q_{m_{i}}$ we have $q_{i}(\tau)=Q_{m_{i}}$ because $\mu_{i}\left(Q_{m_{i}}\right) Q_{m_{i}}=\rho_{m_{i}}$, and $q_{i}(T)<Q_{m_{i}}$ because $\dot{q}_{i}=$ $-\mu_{i}\left(q_{i}\right) q_{i}<0$. With $q_{i}(0)=Q_{m_{i}}$ we obtain $q_{i}(T)<q_{i}(0)$.
Thus, by continuity of $q_{i}(T)$ with regard to the initial condition, there exists an initial condition $q_{i}(0)=\bar{q}_{i}(0)$ such that $\bar{q}_{i}(T)=\bar{q}_{i}(0)$ and $q_{i}$ is $T$-periodic under control (15).

We can also show that $V_{i}(t)=\left|q_{i}(t)-\bar{q}_{i}(t)\right|$ is decreasing along the solutions : $\dot{V}_{i}(t)=\operatorname{sign}\left(q_{i}(t)-\bar{q}_{i}(t)\right)\left(\dot{q}_{i}(t)-\right.$ $\left.\dot{\bar{q}}_{i}(t)\right)<0$ because $\operatorname{sign}\left(\dot{q}_{i}-\dot{\bar{q}}_{i}\right)=\operatorname{sign}\left(-\mu_{i}\left(q_{i}\right) q_{i}+\right.$ $\left.\mu_{i}\left(\bar{q}_{i}\right) \bar{q}_{i}\right)=-\operatorname{sign}\left(q_{i}-\bar{q}_{i}\right) . V_{i}$ being a Lyapunov function converging to zero, $\lim _{t \rightarrow+\infty} q_{i}(t)=\bar{q}_{i}(t)$.

Therefore, with control $(15), \bar{q}_{i}(t)$ attracts all solutions $q_{i}(t), q_{i}$ converges towards a $T$-periodic behavior, and so does $\mu_{i}\left(q_{i}(t)\right)$. We will see in section 5.2 that this periodic behavior, caused by the periodic stresses, can lead us to a new competitiveness criterion.

\section{SIMULATIONS WITH THREE SPECIES}

These simulations have been carried out on the Droop model (4) with control (11) so that the total biomass concentration converge toward $X_{T}^{\star}$.

We have used Michaelis-Menten absorption rates $\rho_{i}(s)$ and Droop's growth rates $\mu_{i}\left(q_{i}\right)$.

$$
\begin{aligned}
\rho_{i}(s) & =\rho_{m_{i}} \frac{s}{s+K_{s_{i}}} \\
\mu_{i}\left(q_{i}\right) & =\bar{\mu}_{i}\left(1-\frac{K_{q_{i}}}{q_{i}}\right)
\end{aligned}
$$

And the following parameters for the species, whose approximate values come from I. Vatcheva, H. de Jong, O. Bernard, N. J.I. Mars [2006] :

\begin{tabular}{|c||c|c|}
\hline species & $K_{s}(\mu \mathrm{mol} / L)$ & $\rho_{m}\left(10^{-9} \cdot\left(\mu \mathrm{mol} /(\mu \mathrm{m})^{3}\right) / \mathrm{day}\right)$ \\
\hline \hline 1 & 0.05 & 9 \\
\hline 2 & 0.01 & 14 \\
\hline 3 & 0.15 & 8 \\
\hline
\end{tabular}

\begin{tabular}{|c||c|c|}
\hline species & $K_{q}\left(10^{-9} \cdot \mu \mathrm{mol} /(\mu \mathrm{m})^{3}\right)$ & $\bar{\mu}(1 /$ day $)$ \\
\hline \hline 1 & 1.5 & 1.5 \\
\hline 2 & 5 & 6 \\
\hline 3 & 2 & 3 \\
\hline
\end{tabular}

\begin{tabular}{|c||c|c|}
\hline species & $\mu_{m}(1 /$ day $)$ & $\mu_{m} \cdot Q_{m} / K_{q}(1 /$ day $)$ \\
\hline \hline 1 & 1.2 & 6 \\
\hline 2 & 1.91 & 2.8 \\
\hline 3 & 1.71 & 4 \\
\hline
\end{tabular}

\subsection{Selection of the biggest $\mu_{m_{i}}$}

We have used a big constant $s_{i n}=10 \mu \mathrm{mol} / \mathrm{L}$ and a small $X_{T}^{\star}=1 \cdot 10^{9} \cdot(\mu m)^{3} / L$, as was previously advised in section 3.3. We have thus obtained $\dot{s} \approx D\left(s_{\text {in }}-s\right)$ and $s$ converges to $s_{\text {in }}$ with $\mu_{i}\left(Q_{i}\left(s_{i n}\right)\right) \approx \mu_{m_{i}}$.

Figure 2 shows the result of the simulation, where species 2 with biggest $\mu_{m_{i}}$ excludes all others. 


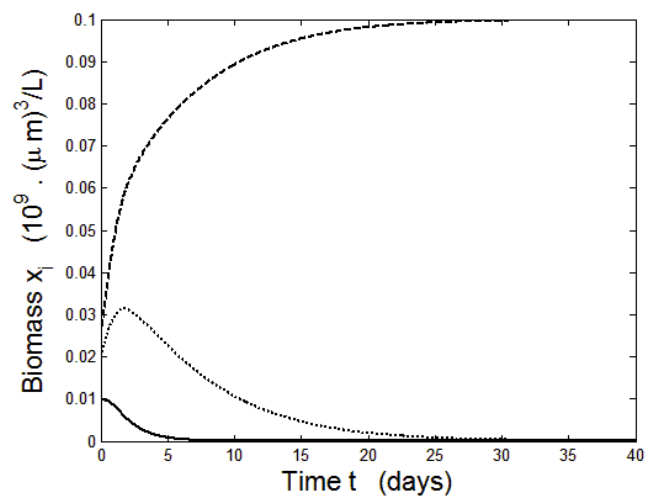

Fig. 2. Simulation of the selection of the species with biggest $\mu_{m_{i}}$

Lines \& species : Solid-1, Dashed-2, Dotted-3

\subsection{Selection under periodic substrate stresses}

For these simulations we have used controls (16) and (17) to approximate ideal control (15), with $T=1$ day, $X_{T}^{\star}=400 \cdot 10^{9}(\mu m)^{3} / L$, and $X_{T}(0)=220 \cdot 10^{9}(\mu m)^{3} / L$

For the rising phase we have used $s_{M}=5 \mu \mathrm{mol} / L$ with $s_{M} \gg K_{s_{i}}$ so that $\rho_{i}\left(s_{M}\right) \approx \rho_{m_{i}}$, and $\lambda=1000$ so that $s$ rises fast.

For the falling phase we have used $\gamma=100 \cdot 10^{9}(\mu m)^{3} / L<$ $\min \left(X_{T}(0), X_{T}^{\star}\right)$, and $\phi(s)=\rho_{m_{\phi}} \frac{s}{s+K_{s_{\phi}}}$ with $\rho_{m_{\phi}}=5$. $10^{9} \mu \mathrm{mol} /(\mu \mathrm{m})^{3}<\rho_{m_{i}}$ and $K_{s_{\phi}}=1 \mu \mathrm{mol} / L>K_{s_{i}}$ so that $\phi(s)<\rho_{i}(s) \quad \forall i \in\{1, \ldots, N\}, \forall s$. In section $4 \mathrm{We}$ have obtained $\dot{s}=-\gamma \phi(s)$. The fall is thus equivalent to the consumption of the substrate by a virtual species with absorption parameters $\rho_{m_{\phi}}$ and $K_{s_{\phi}}$, and with biomass concentration $\gamma$.

We know how to predict $\tau_{\text {fall }}$, until $s$ reaches a small value $s_{0}=0.005 \mu \mathrm{mol} / L$ :

$\frac{d s}{d t}=-\gamma \rho_{m_{\phi}} \frac{s}{s+K_{s_{\phi}}}$ leads us to $d t=-\frac{1}{\gamma \rho_{m_{\phi}}}\left(1+\frac{K_{s_{\phi}}}{s}\right) d s$ and to $\tau_{\text {fall }}=\frac{1}{\gamma \rho_{m_{\phi}}}\left(s_{M}-s_{0}+K_{s_{\phi}} \ln \left(\frac{s_{M}}{s_{0}}\right)\right)$

It is thus possible to choose $\tau_{\text {rise }}$ so that $\tau=\tau_{\text {fall }}+\tau_{\text {rise }}$, and to approach control (15).

With (18) and with functions (19), after some algebraic calculus, we have demonstrated that the internal substrate storages $q_{i}$ converge towards $T$-periodic functions :

$\bar{q}_{i}(t)=\left\{\begin{array}{l}Q_{m_{i}}\left(1-e^{-\bar{\mu}_{i} t}\right)+\bar{q}_{i}(0) e^{-\bar{\mu}_{i} t} \text { if } 0 \leq t \equiv T \leq \tau \\ K_{q_{i}}\left(1-e^{-\bar{\mu}_{i}(t-\tau)}\right)+\bar{q}_{i}(\tau) e^{-\bar{\mu}_{i}(t-\tau)} \text { else }\end{array}\right.$

with $\bar{q}_{i}(0)=Q_{m_{i}}-\left(Q_{m_{i}}-K_{q_{i}}\right) \frac{1-e^{-\bar{\mu}_{i}(T-\tau)}}{1-e^{-\bar{\mu}_{i} T}}$

and $\bar{q}(\tau)=K_{q_{i}}+\left(Q_{m_{i}}-K_{q_{i}}\right) \frac{1-e^{-\bar{\mu}_{i} \tau}}{1-e^{-\bar{\mu}_{i} T}}$

with $\lim _{t \rightarrow+\infty} q_{i}(t)=\bar{q}_{i}(t)$ : the growth rate functions $\mu_{i}\left(q_{i}(t)\right)$ become $T$-periodic.

We have then calculated :

$\mu_{\text {mean }_{i}}=\frac{1}{T} \frac{\mu_{m_{i}}}{\bar{\mu}_{i}} \ln \left(1+\frac{Q_{m_{i}} / K_{q_{i}}}{\frac{1}{1-e^{-\bar{\mu}_{i} \tau}}+\frac{\left(Q_{m_{i}} / K_{q_{i}}\right) e^{-\bar{\mu}_{i} T}-1}{1-e^{-\bar{\mu}_{i} T}}}\right)$
Criterion 6. Periodic stresses' Competitiveness Criterion Under control (15),

with $\tau \approx T$ the species with biggest $\mu_{\text {mean }} \approx \mu_{m_{i}}$ excludes all others from the chemostat;

with $\tau \ll T$ and $\tau \ll \frac{1}{\bar{\mu}_{i} \cdot Q_{m_{i}} / K_{q_{i}}}$ the species with biggest $\frac{T}{\tau} \mu_{\text {mean }_{i}} \approx \mu_{m_{i}} \frac{Q_{m_{i}}}{K_{q_{i}}}$ excludes all others from the chemostat.

where $\frac{Q_{m_{i}}}{K_{q_{i}}}$ represents the capacity of the species to increase its internal substrate storage.

Figure 3 shows that different species can be selected with different environmental condition $\tau$, because for each species $i$ here, there exists a $\tau$ value such that $\mu_{\text {mean }_{i}}$ is higher than the other species' mean growth.

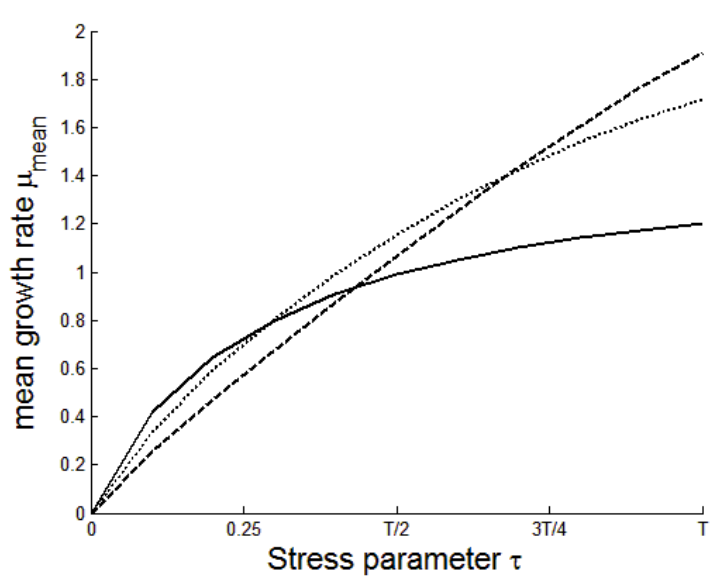

Fig. 3. $\mu_{\text {mean }_{i}}(\tau)$ for the three species. The species with biggest $\mu_{\text {mean }_{i}}$ will be selected. It is thus possible to select each of the three secies with different $\tau$ Lines \& species : Solid-1, Dashed-2, Dotted-3

Finally, Figure 4 presents the simulations.

\section{CONCLUSION}

This work shows that it is in theory possible to select species exhibiting a desired feature in the chemostat. Moreover the selection criterion, which is the maximum growth rate under given environmental conditions, seems promising for various biotechnological applications.

The selection occurs because the total biomass concentration $X_{T}=\sum_{i=1}^{N} x_{i}$ is upper bounded. It is possible to bound $X_{T}$ by other means than controlling $D$ (for instance with the light). It is also most certainly possible to find new (20)selection criteria by changing the environmental conditions with $D, s_{i n}$, and with other controls such as an inhibitor's concentration.

This selection method makes it possible to select quite easily a species for its study or culture. It could be used to identify species which grow in hostile conditions, to amplify and verify the presence of a species in a medium, or to select a species which is interesting for a specific biotechnological objective.

This work could be extended to models describing predation or parasitism by other types of microorganisms, or 

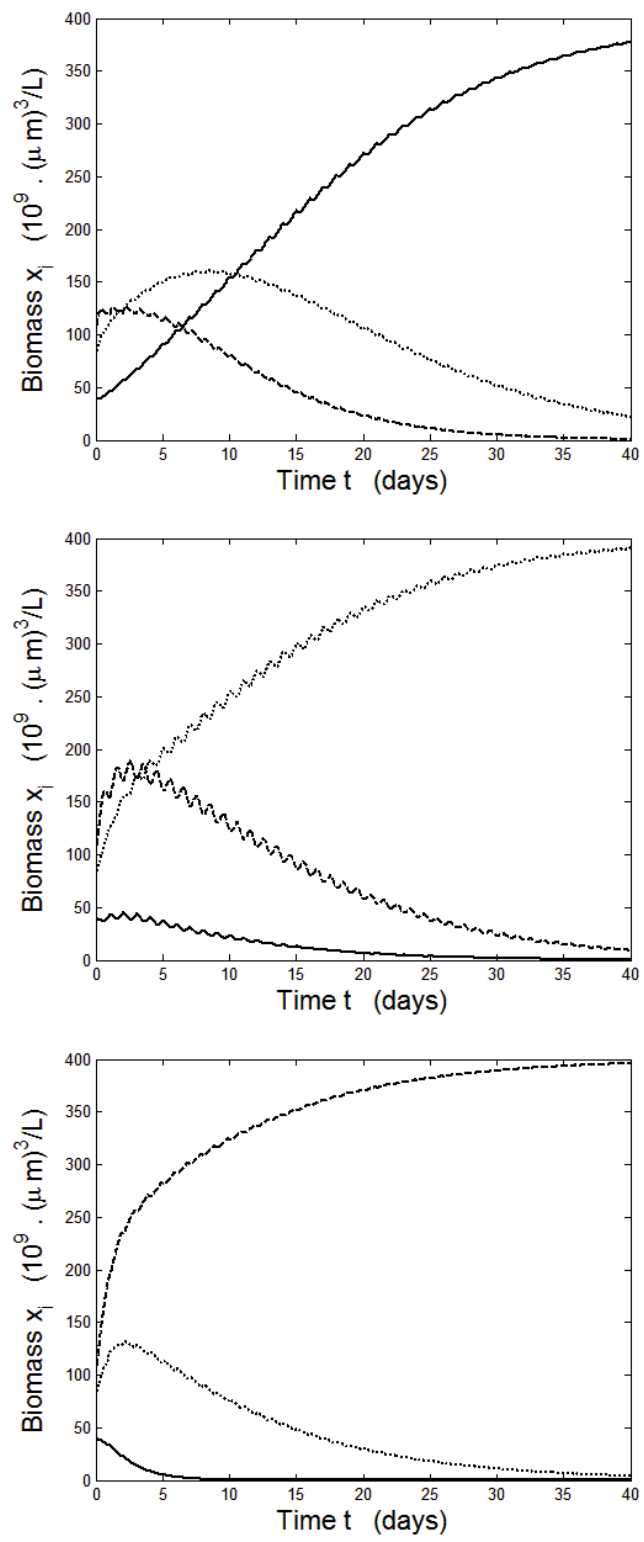

Fig. 4. Simulations of competition in the Droop model, under controls (11), (16) and (17).

top : $\tau=0.1 T$, middle $: \tau=0.5 T$, bottom $: \tau=0.9 T$

Lines \& species : Solid-1, Dashed-2, Dotted-3

agressive interactions between the species such as the production of a chemical which inhibates other species growth (see A.G. Fredrickson and G. Stephanopoulos [1981] for a presentation of factors which can mitigate the severity of the competition).

\section{ACKNOWLEDGEMENTS}

This work was carried out as part of the APPLE project, and financed by the French national program PROOF.

\section{REFERENCES}

S.R. Hansen and S.P. Hubell Single-nutrient microbial competition: qualitative agreement between experimental and theoretically forecast outcomes Science, 207(4438): 1491-1493, 1980
A.G. Fredrickson and G. Stephanopoulos Microbial competition Science, 213: 1491-1493, 1981

S.W. Brown and S.G. Oliver Isolation of ethanol-tolerant mutants of yeast by continuous selection European Journal of Applied Microbiology and Biotechnology, 16: $119-122,1982$

H.L. Smith and P. Waltman The theory of the chemostat. Dynamics of microbial competition Cambridge Studies in Mathematical Biology. Cambridge University Press, 1995

J. Gouzé and G. Robledo Feedback control for nonmonotone competition models in the chemostat Nonlinear Analysis: Real World Applications, 6: 671-690, 2005

P. de Leenheer, B. Li, and H.L. Smith Competition in the chemostat : some remarks Canadian applied mathematics quarterly, 11(2): 229-247, 2003

N.S. Rao and E.O. Roxin Controled growth of competing species SIAM, Journal on Applied Mathematics, 50(3): 853-864, 1990

J. Passarge, S. Hol, M. Escher, and J. Huisman Competition for nutrients and light: Stable coexistence, alternative stable states, or competitive exclusion? Ecological Monographs, 55(1): 55-72, 2006

R. Arditi and H. Saiah Empirical-evidence of the role of heterogeneity in ratio-dependent consumption Ecology, 73(5): 1544-1551, 1992

B.J.M. Bohannan and R.E. Lenski Linking genetic change to community evolution: insights from studies of bacteria and bacteriophage Ecology Letters, 3(4): 362-377, 2000

H. Ducobu, J. Huisman, R.R. Jonker, and L.R. Mur Competition between a prochlorophyte and a cyanobacterium under various phosphorus regimes: Comparison with the Droop model Journal of Phycology, 34(3): 467476

S.S. Pilyugin, G.T. Reeves, and A. Narang Predicting stability of mixed microbial cultures from single species experiments Mathematical Biosciences 192(2): 85-109, 2004

C. Lobry and J. Harmand A new hypothesis to explain the coexistence of $\mathrm{n}$ species in the presence of a single resource Comptes Rendus Biologies 329(1): 40-46, 2006

C. Lobry, F. Mazenc, and A. Rapaport Persistence in ecological models of competition for a single resource Comptes Rendus Mathematique 340(3): 199-204, 2005

Juan Jimnez and Taha Bentez Selection of ethanoltolerant yeast hybrids in pH-regulated continuous culture Appl Environ Microbiology, 54(4): 917-922, 1988

J. Hesseler, J.K. Schmidt, U. Reichl, D. Flockerzi Coexistence in the chemostat as a result of metabolic byproducts Journal of Mathematical Biology, 53(4): 556584,2006

B.T. Li , H.L. Smith Periodic coexistence of four species competing for three essential resources Mathematical Biosciences, 184(2): 115-135, 2003

O. Bernard, G. Malara, and A. Sciandra The effects of a controlled fluctuating nutrient environment on continuous cultures of phytoplankton monitored by a computer J. Exp. Mar. Biol. Ecol, 197:263-278, 1996

I. Vatcheva, H. de Jong, O. Bernard, N. J.I. Mars Experiment selection for the discrimination of semiquantitative models of dynamical systems Artificial Intelligence, 170(4-5), 472-506, 2006 